\title{
Some Approximation Properties of the Generalized Baskakov operators
}

\author{
Prashantkumar Patel ${ }^{\mathrm{a}, \mathrm{b}, 1}$, Vishnu Narayan Mishra ${ }^{\mathrm{a}, \mathrm{d}}$, Mediha Örkcü ${ }^{\mathrm{c}}$ \\ ${ }^{a}$ Department of Applied Mathematics \& Humanities, S. V. National Institute of Technology, Surat-395 007 (Gujarat), India \\ ${ }^{b}$ Department of Mathematics, St. Xavier's College(Autonomous), Ahmedabad-380 009 (Gujarat), India \\ ${ }^{c}$ Department of Mathematics, Faculty of Sciences, Gazi University, Teknikokullar, 06500 Ankara, Turkey \\ ${ }^{d}$ L. 1627 Awadh Puri Colony Beniganj, Phase-III, Opposite - Industrial Training Institute (ITI), Ayodhya Main Road, Faizabad, \\ Uttar Pradesh 224 001, India
}

\begin{abstract}
The present paper deals with a generalization of the Baskakov operators. Some direct theorems, asymptotic formula and $A$-statistical convergence are established. Our results are based on a $\rho$ function. These results include the preservation properties of the classical Baskakov operators.

Keywords: asymptotic analysis, convergence analysis, convergence rate, The Baskakov operators, $A$-statistical convergence

2000 Mathematics Subject Classification: 41A25, 41A36, Secondary 47B33, 47B38.
\end{abstract}

\section{Introduction}

In [2], Baskakov discussed the following positive linear operators on the unbounded interval $[0, \infty)$,

$$
V_{n}(f ; x)=\sum_{k=0}^{\infty} v_{k, n}(x) f\left(\frac{k}{n}\right), \quad x \in[0, \infty), n \in \mathbf{N},
$$

where $f$ is an appropriate function defined on the unbounded interval $[0, \infty)$, for which the above series is convergent and $v_{n, k}(x)=\left(\begin{array}{c}n+k-1 \\ k\end{array}\right) \frac{x^{k}}{\left(1+x \eta^{+k}\right.}$.

In 2011, Cárdenas-Morales et al. 3] introduced a generalized Bernstein operators by fixing $e_{0}$ and $e_{1}$, given by

$$
L_{n}(f ; x)=\sum_{k=0}^{n}\left(\begin{array}{l}
n \\
k
\end{array}\right) x^{2 k}\left(1-x^{2}\right)^{n-k} f\left(\sqrt{\frac{k}{n}}\right), \quad x \in[0,1], n \in \mathbf{N},
$$

where $f \in C[0,1]$. This is a special case of the operators $B_{n}^{\tau} f=B_{n}\left(f \circ \tau^{-1}\right) \circ \tau$, for $\tau=e_{2}$, where $B_{n}$ is the classical Bernstein operators.

Consider a real valued function $\rho$ on $[0, \infty)$ satisfied following two conditions:

1. $\rho$ is a continuously differentiable function on $[0, \infty)$,

2. $\rho(0)=0$ and $\inf _{x \in[0, \infty)} \rho^{\prime}(x) \geq 1$.

Email addresses: prashant225@gmail.com (Prashantkumar Patel), vishnu_narayanmishra@yahoo.co.in; vishnunarayanmishra@gmail.com (Vishnu Narayan Mishra), medihaakcay@gazi.edu.tr (Mediha Örkcü)

${ }^{1}$ Corresponding authors 
Throughout the manuscript, we denote the above two conditions as $c_{1}$ and $c_{2}$. Recently, In [1] the following generalization of Szász-Mirakyan operators are constructed,

$$
S_{n}^{\rho}(f ; x)=\exp (-n \rho(x)) \sum_{k=0}^{\infty}\left(f \circ \rho^{-1}\right)\left(\frac{k}{n}\right) \frac{(n \rho(x))^{k}}{k !}, \quad x \in[0,1], n \in \mathbf{N} .
$$

Notice that if $\rho=e_{1}$ then the operators (1.3) reduces to the well known Szász-Mirakyan operators. Aral et al. [1], gave quantitative type theorems in order to obtain the degree of weighted convergence with the help of a weighted modulus of continuity constructed using the function $\rho$ of the operators (1.3). Very recently, some researchers have discussed shape preserving properties of the generalized Bernstein, Baskakov and Szász-Mirakjan operators in [17-20].

\section{Construction of the Operators}

This motivated us to generalize the Baskakov operators (1.1) as

$$
\begin{aligned}
V_{n}^{\rho}(f ; x) & =\sum_{k=0}^{\infty}\left(f \circ \rho^{-1}\right)\left(\frac{k}{n}\right)\left(\begin{array}{c}
n+k-1 \\
k
\end{array}\right) \frac{(\rho(x))^{k}}{(1+\rho(x))^{n+k}} \\
& =\left(V_{n}^{\rho}\left(\left(f \circ \rho^{-1}\right) \circ \rho\right)\right)(x) \\
& =\sum_{k=0}^{\infty} f\left(\rho^{-1}\left(\frac{k}{n}\right)\right) v_{\rho, n, k}(x),
\end{aligned}
$$

where $n \in \mathbf{N}, x \in[0, \infty)$ and $\rho$ is a function defined as in conditions $q_{1}$ and $q_{2}$,

Observe that, $V_{n}^{\rho}(f ; x)=V_{n}(f ; x)$ if $\rho=e_{1}$. In fact, direct calculation gives that

$$
\begin{gathered}
V_{n}^{\rho}\left(e_{0} ; x\right)=1 ; \\
V_{n}^{\rho}(\rho ; x)=\rho(x) ; \\
V_{n}^{\rho}\left(\rho^{2} ; x\right)=\rho^{2}(x)+\frac{\rho^{2}(x)+\rho(x)}{n} .
\end{gathered}
$$

In this manuscript, we are dealing with approximation properties the operators (2.1). In the next section, we establish some direct results using generalized modulus of continuity. The Voronovskaya Asymptotic formula and $A$-Statistical convergence of the operators $V_{n}^{\rho}$ are discuss in Section 4 and 5.

\section{Direct Theorems}

Consider $\phi^{2}(x)=1+\rho^{2}(x)$. Notice that, $\lim _{x \rightarrow \infty} \rho(x)=\infty$ because the condition 2 . Denote $B_{\phi}([0, \infty))$ as set of all real valued function on $[0, \infty)$ such that $|f(x)| \leq M_{f} \phi(x)$, for all $x \in[0, \infty)$, where $M_{f}$ is a constant

depending on $f$. Observe that, $B_{\phi}([0, \infty))$ is norm linear space with the norm $\|f\|_{\phi}=\sup \left\{\frac{|f(x)|}{\phi(x)}: x \in[0, \infty)\right\}$. Also, $C_{\phi}([0, \infty))$ as the set all continuous function in $B_{\phi}([0, \infty))$ and

$$
C_{\phi}^{k}([0, \infty))=\left\{f \in C_{\phi}([0, \infty)): \lim _{x \rightarrow \infty} \frac{f(x)}{\phi(x)}=k_{f}, k_{f} \text { is constant depaneding on } f\right\} .
$$


Let $U_{\phi}([0, \infty))$ be the space of functions $f \in C_{\phi}([0, \infty))$, such that $\frac{f(x)}{\phi(x)}$ is uniformly continuous. Also, $C_{\phi}^{k}([0, \infty)) \subset$ $U_{\phi}([0, \infty)) \subset C_{\phi}([0, \infty)) \subset B_{\phi}([0, \infty))$.

Lemma 1. [11] The positive linear operators $L_{n}: C_{\phi}([0, \infty)) \rightarrow B_{\phi}([0, \infty))$ for all $n \geq 1$ if and only if the inequality

$$
\left|L_{n}(\phi ; x)\right| \leq K_{n} \phi(x), \quad x \in[0, \infty), n \geq 1,
$$

holds, where $K_{n}$ is a positive constant.

Theorem 1. [11] Let the sequence of linear positive operators $\left(L_{n}\right)_{n \geq 1}, L_{n}: C_{\phi}([0, \infty)) \rightarrow B_{\phi}([0, \infty))$ satisfy the three conditions

$$
\lim _{n \rightarrow \infty}\left\|L_{n} \rho^{i}-\rho^{i}\right\|=0, \quad i=0,1,2 .
$$

Then

$$
\lim _{n \rightarrow \infty}\left\|L_{n} f-f\right\|=0
$$

for any $f \in C_{\phi}^{k}([0, \infty))$.

By (2.2), (2.4) and Lemma 1, $V_{n}^{\rho}$ is linear positive operators from $C_{\phi}([0, \infty))$ to $B_{\phi}([0, \infty))$.

Theorem 2. For each function $f \in C_{\phi}^{k}([0, \infty))$, we have

$$
\lim _{n \rightarrow \infty}\left\|V_{n}^{\rho}(f ; \cdot)-f\right\|_{\phi}=0
$$

Proof: From (2.2) and (2.3), we write

$$
\left\|V_{n}^{\rho}(1 ; \cdot)-1\right\|_{\phi}=0 \text { and }\left\|V_{n}^{\rho}(\rho ; \cdot)-\rho\right\|_{\phi}=0
$$

Also,

$$
\left\|V_{n}^{\rho}\left(\rho^{2} ; \cdot\right)-\rho^{2}\right\|_{\phi}=\sup _{x \in[0, \infty)} \frac{\rho^{2}(x)+\rho(x)}{n\left(1+\rho^{2}(x)\right)} \leq \frac{2}{n}
$$

Therefore, we have

$$
\left\|V_{n}^{\rho}\left(\rho^{i} ; \cdot\right)-\rho^{i}\right\|_{\phi} \rightarrow 0 \text { as } n \rightarrow \infty, \text { for } i=0,1,2 .
$$

Hence by Theorem 1, the equation (3.1) is also true.

In 13] the following weighted modulus of continuity is defined

$$
\omega_{\rho}(f ; \delta):=\omega(f ; \delta)_{[0, \infty)}=\sup _{\substack{x, t \in[0, \infty) \\|\rho(x)-\rho(t)| \leq \delta}} \frac{|f(t)-f(x)|}{|\phi(t)-\phi(x)|}
$$

for each $f \in C_{\phi}([0, \infty))$ and for every $\delta>0$.

We call the function $\omega_{\rho}(f ; \delta)$ weighted modulus of continuity. We observe that $\omega_{\rho}(f ; 0)=0$ for every $f \in C_{\phi}([0, \infty))$ and that the function $\omega_{\rho}(f ; \delta)$ is nonnegative and nondecreasing with respect to $\delta$ for $f \in C_{\phi}([0, \infty))$. Here, we 
consider the spaces $C_{\phi}^{k}([0, \infty)), U_{\phi}([0, \infty)), C_{\phi}([0, \infty))$ and $B_{\phi}([0, \infty))$ having the conditions $\mathbb{1}_{1}$ and $q_{2}$ Under these conditions, $|x-t| \leq|\rho(x)-\rho(t)|$, for every $x, t \in[0, \infty)$ is true.

Lemma 2. [1]] $\lim _{\delta \rightarrow 0} \omega_{\rho}(f ; \delta)=0$, for every $f \in U_{\phi}([0, \infty))$.

Theorem 3. [13] Let $L_{n}: C_{\phi}([0, \infty)) \rightarrow B_{\phi}([0, \infty))$ be a sequence of positive linear operators with

$$
\begin{aligned}
\left\|L_{n}\left(\rho^{0}\right)-\rho^{0}\right\|_{\phi^{0}} & =a_{n}, \\
\left\|L_{n}(\rho)-\rho\right\|_{\phi^{\frac{1}{2}}} & =b_{n}, \\
\left\|L_{n}\left(\rho^{2}\right)-\rho^{2}\right\|_{\phi} & =c_{n}, \\
\left\|L_{n}\left(\rho^{3}\right)-\rho^{3}\right\|_{\phi^{\frac{3}{2}}} & =d_{n},
\end{aligned}
$$

where $a_{n}, b_{n}, c_{n}$ and $d_{n}$ tends to zero as $n \rightarrow \infty$. Then

$$
\left\|L_{n}(f)-f\right\|_{\phi^{\frac{3}{2}}}=\left(7+4 a_{n}+2 c_{n}\right) \omega_{\rho}\left(f ; \delta_{n}\right)+a_{n}\|f\|_{\phi}
$$

for all $f \in C_{\phi}([0, \infty))$, where

$$
\delta_{n}=2 \sqrt{\left(a_{n}+2 b_{n}+c_{n}\right)\left(1+a_{n}\right)}+a_{n}+3 b_{n}+3 c_{n}+d_{n} .
$$

Theorem 4. For all $f \in C_{\phi}([0, \infty))$, we have

$$
\left\|V_{n}^{\rho}(f ; \cdot)-f\right\|_{\phi^{\frac{3}{2}}} \leq\left(7+\frac{4}{n}\right) \omega_{\rho}\left(f, \frac{2 \sqrt{2}}{\sqrt{n}}+\frac{16}{n}\right) .
$$

Proof: Notice that

$$
\left\|V_{n}^{\rho}\left(\rho^{0} ; \cdot\right)-\rho^{0}\right\|_{\phi^{0}}=0=a_{n}
$$

and

$$
\left\|V_{n}^{\rho}(\rho ; \cdot)-\rho\right\|_{\phi^{\frac{1}{2}}}=0=b_{n}
$$

Form equation (3.2), we have

$$
c_{n}=\left\|V_{n}^{\rho}\left(\rho^{2} ; \cdot\right)-\rho^{2}\right\|_{\phi} \leq \frac{2}{n} .
$$

Now,

$$
V_{n}^{\rho}\left(\rho^{3} ; x\right)=\frac{\rho(x)}{n^{2}}+\frac{3(1+n) \rho(x)^{2}}{n^{2}}+\frac{\left(2+3 n+n^{2}\right) \rho(x)^{3}}{n^{2}} .
$$


We can write

$$
\begin{aligned}
d_{n}=\left\|V_{n}^{\rho}\left(\rho^{3} ; \cdot\right)-\rho^{3}\right\|_{\phi^{\frac{3}{2}}}= & \sup _{x \in[0, \infty)}\left\{\frac{\rho(x)}{n^{2}\left(1+\rho^{2}(x)\right)^{\frac{3}{2}}}\right. \\
& \left.+\frac{3(1+n) \rho(x)^{2}}{n^{2}\left(1+\rho^{2}(x)\right)^{\frac{3}{2}}}+\frac{(2+3 n) \rho(x)^{3}}{n^{2}\left(1+\rho^{2}(x)\right)^{\frac{3}{2}}}\right\} \\
\leq & \frac{1}{n}+\frac{4}{n}+\frac{5}{n}=\frac{10}{n} .
\end{aligned}
$$

Observe that, the condition (3.4)-(3.7) are satisfied, therefore by theorem 3, we have

$$
\left\|V_{n}^{\rho}(f ; \cdot)-f\right\|_{\phi^{\frac{3}{2}}} \leq\left(7+\frac{4}{n}\right) \omega_{\rho}\left(f, \frac{2 \sqrt{2}}{\sqrt{n}}+\frac{16}{n}\right) .
$$

This completes the proof of Theorem 4

Theorem 5. For all $f \in U_{\phi}^{k}([0, \infty))$, we have $\lim _{n \rightarrow \infty}\left\|V_{n}^{\rho}(f ; \cdot)-f\right\|_{\phi^{\frac{3}{2}}}=0$.

The proof follows from the Theorem 4 and Lemma 2 .

\section{Voronovskaya Asymptotic formula}

Now we give the following Voronovskaya type theorem. We use the technique developed in [1, 3$]$.

Theorem 6. Let $f \in C[0, \infty), x \in[0, \infty)$ and suppose that the first and second derivatives of $f \circ \rho^{-1}$ exist at $\rho(x)$. If the second derivative of $f \circ \rho^{-1}$ is bounded on $[0, \infty)$ then we have

$$
\lim _{n \rightarrow \infty} n\left(V_{n}^{\rho}(f ; x)-f(x)\right)=\frac{1}{2} \rho(x)(1+\rho(x))\left(f \circ \rho^{-1}\right)^{\prime \prime}(\rho(x))
$$

Proof: By the Taylor expansion of $f \circ \rho^{-1}$ at the point $\rho(x) \in[0, \infty)$, there exists $\xi$ lying between $x$ and $t$ such that

$$
\begin{aligned}
f(t)= & \left(f \circ \rho^{-1}\right)(\rho(t))=\left(f \circ \rho^{-1}\right)(\rho(x))+\left(f \circ \rho^{-1}\right)^{\prime}(\rho(x))(\rho(t)-\rho(x)) \\
& +\frac{1}{2}\left(f \circ \rho^{-1}\right)^{\prime \prime}(\rho(x))(\rho(t)-\rho(x))^{2}+\lambda_{x}(t)(\rho(t)-\rho(x))^{2},
\end{aligned}
$$

where

$$
\lambda_{x}(t)=\frac{\left(f \circ \rho^{-1}\right)^{\prime}(\rho(\xi))-\left(f \circ \rho^{-1}\right)^{\prime \prime}(\rho(x))}{2} .
$$

Note that, the assumptions on $f$ together with definition (4.3) ensure that $\left|\lambda_{x}(t)\right| \leq M$ for all $t$ and converges to zero as $t \rightarrow x$.

Applying the operators (2.1) to the above equation (4.2) equality, we get

$$
\begin{aligned}
V_{n}^{\rho}(f ; x)-f(x)= & \left(f \circ \rho^{-1}\right)^{\prime}(\rho(x)) V_{n}^{\rho}((\rho(t)-\rho(x)) ; x) \\
& +\frac{1}{2}\left(f \circ \rho^{-1}\right)^{\prime \prime}(\rho(x)) V_{n}^{\rho}\left((\rho(t)-\rho(x))^{2} ; x\right) \\
& +V_{n}^{\rho}\left(\lambda_{x}(t)(\rho(t)-\rho(x))^{2} ; x\right),
\end{aligned}
$$


by equations (2.2), (2.3) and (2.4), we have

$$
\begin{gathered}
\lim _{n \rightarrow \infty} n V_{n}^{\rho}((\rho(t)-\rho(x)) ; x)=0 ; \\
\lim _{n \rightarrow \infty} n V_{n}^{\rho}\left((\rho(t)-\rho(x))^{2} ; x\right)=\rho(x)(1+\rho(x)) .
\end{gathered}
$$

Therefore,

$$
\begin{aligned}
\lim _{n \rightarrow \infty} n\left(V_{n}^{\rho}(f ; x)-f(x)\right)= & \frac{1}{2} \rho(x)(1+\rho(x))\left(f \circ \rho^{-1}\right)^{\prime \prime}(\rho(x)) \\
& +\lim _{n \rightarrow \infty} n\left(V_{n}^{\rho}\left(\lambda_{x}(t)(\rho(t)-\rho(x))^{2} ; x\right)\right) .
\end{aligned}
$$

Now we estimate the last term on the right hand side of the above equality. Let $\epsilon>0$ and choose $\delta>0$ such that $\left|\lambda_{x}(t)\right|<\epsilon$ for $|t-x|<\delta$. Also it is easily seen that by condition q2,$|\rho(t)-\rho(x)|=\rho(\eta)|t-x| \geq|t-x|$. Therefore, if $|\rho(t)-\rho(x)|<\delta$, then $\left|\lambda_{x}(t)(\rho(t)-\rho(x))^{2}\right|<\epsilon(\rho(t)-\rho(x))^{2}$, while if $|\rho(t)-\rho(x)| \geq \delta$, then since $\left|\lambda_{x}(t)\right| \leq M$ we have $\left|\lambda_{x}(t)(\rho(t)-\rho(x))^{2}\right| \leq \frac{M}{\delta^{2}}(\rho(t)-\rho(x))^{4}$. So we can write

$$
\begin{aligned}
V_{n}^{\rho}\left(\lambda_{x}(t)(\rho(t)-\rho(x))^{2} ; x\right)< & \epsilon\left(V_{n}^{\rho}\left((\rho(t)-\rho(x))^{2} ; x\right)\right) \\
& +\frac{M}{\delta^{2}}\left(V_{n}^{\rho}\left((\rho(t)-\rho(x))^{4} ; x\right)\right) .
\end{aligned}
$$

Direct calculations show that

$$
V_{n}^{\rho}\left((\rho(t)-\rho(x))^{4} ; x\right)=O\left(\frac{1}{n^{2}}\right)
$$

Hence

$$
\lim _{n \rightarrow \infty} n V_{n}^{\rho}\left(\lambda_{x}(t)(\rho(t)-\rho(x))^{2} ; x\right)=0,
$$

which completes the proof of the Theorem 8 .

\section{A-Statistical Convergence}

Now, we introduces some notation and the basic definitions, which used in this section. Let $A=\left(a_{i j}\right)$ be an infinite summability matrix. For given sequence $x=\left(x_{n}\right)$, the $A$-transform to $x$, denoted by $A x=\left((A x)_{j}\right)$, is given by $(A x)_{j}=\sum_{n=1}^{\infty} a_{j n} x_{n}$, provided the series converges for each $j$. We say that $A$ is regular, if $\lim _{j}(A x)_{j}=L$ whenever $\lim _{j} x_{j}=L[12]$.

Now, we assume that $A$ is a nonnegative regular summability matrix and $K$ is a subset of $\mathbf{N}$, the set of all natural numbers. The $A$-density of $K$ is defined by $\delta_{A}(K)=\lim _{j} \sum_{n=1}^{\infty} a_{j n} \chi_{K}(n)$ provided the limit exists, where $\chi_{K}$ is the characteristic function of $K$. Then the sequence $x=\left(x_{n}\right)$ is said to be $A$-statistically convergent to the number $L$ if, for every $\epsilon>0, \delta_{A}\left\{n \in \mathbf{N}:\left|x_{n}-L\right| \geq \epsilon\right\}=0$; or equivalently $\lim _{j} \sum_{n:\left|x_{n}-L\right| \geq \epsilon} a_{j n}=0$. We denote this limit by $s t_{A}-\lim x=L[\underline{4}, \underline{5}, \underline{8}, \underline{16}]$.

For the case in which $A=C_{1}$, the Cesàro matrix, $A$-statistical convergence reduces to statistical convergence 7, 9, 10]. Also, taking $A=I$, the identity matrix, $A$-statistical convergence coincides with the ordinary convergence. 
We also note that if $A=\left(a_{j n}\right)$ is a nonnegative regular summability matrix for which $\lim _{j} \max _{n}\left\{a_{j n}\right\}=0$, then $A$ statistical convergence is stronger than convergence [15]. A sequence $x=\left(x_{n}\right)$ is said to be $A$-statistically bounded provided that there exists a positive number $M$ such that $\delta_{A}\left\{n \in \mathbf{N}:\left|x_{n}\right| \leq M\right\}=1$. Recall that $x=\left(x_{n}\right)$ is A-statistically convergent to $\mathrm{L}$ if and only if there exists a subsequence $x_{n(k)}$ of $x$ such that $\delta_{A}\{n(k): k \in \mathbf{N}\}=1$ and $\lim _{k} x_{n(k)}=L($ see $[15,16])$. Note that, the concept of $A$-statistical convergence is also given in normed spaces 14].

In this section, we denote $B_{\phi}([0, \infty))$ by $B_{\phi}$ and $C_{\phi}([0, \infty))$ by $C_{\phi}$. Assume $\phi_{1}(x)$ and $\phi_{2}(x)$ be weight functions satisfying $\lim _{|x| \rightarrow \infty} \frac{\phi_{1}(x)}{\phi_{2}(x)}=0$. If $T$ is a positive operators such that $T: C_{\phi_{1}} \rightarrow B_{\phi_{2}}$, then the operators norm $\|T\|_{C_{\phi_{1}} \rightarrow B_{\phi_{2}}}$ is given by $\|T\|_{C_{\phi_{1}} \rightarrow B_{\phi_{2}}}:=\sup _{\|f\|_{\phi_{1}=1}}\|T f\|_{\phi_{2}}$.

Theorem 7. [6, Thorem 6] Let $A=\left(a_{j n}\right)$ be a non-negative regular summability matrix, let $\left\{T_{n}\right\}$ be a sequence of positive linear operators from $C_{\phi_{1}}$ into $B_{\phi_{2}}$ and assume that $\phi_{1}(x)$ and $\phi_{2}(x)$ be weight functions satisfying $\lim _{|x| \rightarrow \infty} \frac{\phi_{1}(x)}{\phi_{2}(x)}=0$. Then

$$
s t_{A}-\lim _{n}\left\|T_{n} f-f\right\|_{\phi_{2}}=0, \text { for all } f \in C_{\phi_{1}}
$$

if and only if

$$
s t_{A}-\lim _{n}\left\|T_{n} \rho^{v}-\rho^{v}\right\|_{\phi_{1}}=0, \quad v=0,1,2 .
$$

With the help of Theorem 7 we write the following Korovkin type theorem.

Theorem 8. Let $A=\left(a_{j n}\right)$ be a non-negative regular summability matrix, let $\left\{V_{n}\right\}$ be a sequence of positive linear operators from $C_{\phi_{1}}$ into $B_{\phi_{2}}$ as defined in (2.1) and assume that $\phi_{1}(x)$ and $\phi_{2}(x)$ be weight functions satisfying $\lim _{|x| \rightarrow \infty} \frac{\phi_{1}(x)}{\phi_{2}(x)}=0$. Then

$$
s t_{A}-\lim _{n}\left\|V_{n}(f, \cdot)-f\right\|_{\phi_{2}}=0, \text { for all } f \in C_{\phi_{1}} .
$$

Proof: By theorem 7 it is sufficient to prove that,

$$
s t_{A}-\lim _{n}\left\|V_{n}\left(\rho^{v}, \cdot\right)-\rho^{v}\right\|_{\phi_{1}}=0, \quad v=0,1,2 .
$$

It clear that

$$
\left\|V_{n}\left(\rho^{0}, \cdot\right)-\rho^{0}\right\|_{\phi_{1}}=0 \text { and }\left\|V_{n}(\rho, \cdot)-\rho\right\|_{\phi_{1}}=0 .
$$

Hence, equation (5.4) is true for $v=0,1$.

Now, for $v=2$

$$
\left\|V_{n}\left(\rho^{2}, \cdot\right)-\rho^{2}\right\|_{\phi_{1}} \leq \frac{2}{n} .
$$

Due to the equality $s t_{A}-\lim _{n} \frac{1}{n}=0$, the above inequality implies that

$$
s t_{A}-\lim _{n}\left\|V_{n}\left(\rho^{2}, \cdot\right)-\rho^{2}\right\|_{\phi_{1}}=0,
$$

which completes the proof the Theorem 8 


\section{Conclusions}

We constructed sequences of the Baskakov operators which are based on a function $\rho$. This function not only characterizes the operators but also characterizes the Korovkin set $\left\{1, \rho, \rho^{2}\right\}$ in a weighted function space.

Our results include the following: The rate of convergence of these operators to the identity operator on weighted spaces which are constructed using the function $\rho$ and which are subspaces of the space of continuous functions on $[0, \infty)$. We gave quantitative type theorems in order to obtain the degree of weighted convergence with the help of a weighted modulus of continuity constructed using the function $\rho$ and the study of $A$-statistical convergence of the sequence.

\section{References}

[1] A Aral, D Inoan and I Raşa. On the generalized Szász-Mirakyan operators. Results in Mathematics, 65(34):441-452, 2014.

[2] VA Baskakov. An instance of a sequence of linear positive operators in the space of continuous functions. Doklady Akademii Nauk SSSR, 113(2):249-251, 1957.

[3] D Cárdenas-Morales, P Garrancho and I Raşa. Bernstein-type operators which preserve polynomials. Computers $\&$ Mathematics with Applications, 62(1):158-163, 2011.

[4] J Connor. On strong matrix summability with respect to a modulus and statistical convergence. Canad. Math. Bull, 32(2):194-198, 1989.

[5] J Connor and J Kline. On statistical limit points and the consistency of statistical convergence. Journal of mathematical analysis and applications, 197(2):392-399, 1996.

[6] O Duman and C Orhan. Statistical approximation by positive linear operators. Studia Mathematica, 161(2):187-197, 2004.

[7] H Fast. Sur la convergence statistique. In Colloquium Mathematicae, volume 2, pp. 241-244. Institute of Mathematics Polish Academy of Sciences, 1951.

[8] A Freedman and J Sember. Densities and summability. Pacific Journal of Mathematics, 95(2):293-305, 1981.

[9] J Fridy and C Orhan. Statistical limit superior and limit inferior. Proceedings of the American Mathematical Society, 125(12):3625-3631, 1997.

[10] John A Fridy. On statistical convergence. Analysis, 5(4):301-314, 1985.

[11] A. D. Gadžiev. The convergence problem for a sequence of positive linear operators on unbounded sets and theorems analogues to that of P. P. Korovkin. Dokl. Akad. Nauk SSSR, 218:1001-1004, 1974. Also in Soviet Math Dokl. 15, 14331436 (1974) (in English) 
[12] G H Hardy. Divergent Series. Oxford Univ. Press, London, 1949.

[13] A. Holhoş. Quantitative estimates for positive linear operators in weighted space. General Math., 16(4):99-110, 2008.

[14] E Kolk. The statistical convergence in banach spaces. Acta Comm. Univ. Tartuensis, 928:41-52, 1991.

[15] Enno Kolk. Matrix summability of statistically convergent sequences. Analysis, 13(1-2):77-84, 1993.

[16] H I Miller. A measure theoretical subsequence characterization of statistical convergence. Transactions of the American Mathematical Society, 347(5):1811-1819, 1995.

[17] V N Mishra, K Khatri and L N Mishra. Statistical approximation by Kantorovich-type discrete $q$-Beta operators. Advances in Difference Equations, 2013(1):345, 2013.

[18] H Wang, Pu Fagui and Wang K. Shape-preserving and convergence properties for the $q$-Szász-Mirakjan operators for fixed $q \in(0,1)$. Abstract and Applied Analysis, Volume 2014:8 pages, 2014. Article ID 563613

[19] Y Wang and Y Zhou. Shape preserving properties for Bernstein-Stancu operators. Journal of Mathematics, Volume 2014:5 pages, 2014. Article ID 603694

[20] C Zhang and Z Zhu. Preservation properties of the Baskakov-Kantorovich operators. Computers \&5 Mathematics with Applications, 57(9):1450-1455, 2009. 